attribute only of certain races of the organism. Stregulina thinks that those races of bacillus subtilis which come from the soil can be pathogenic. Of twenty-five samples that he inoculated into guinea pigs, sixteen were pathogenic and three of these produced panophthalmitis. On the other hand, such factors as repeated animal passage and cultivation on media containing blood, which have long been known to enhance pothogenicity may play a part in some indirect way. Indeed a pathogenic bacillus subtilis has been obtained by Charrin and De Nittis ${ }^{5}$ by repeated animal passage and cultivation on blood-media. But they found that at least three-quarters of their most pathogenic culture obtained in this way was required in order to kill a guinea pig.

I wish here to thank my chief, Dr. A. A. Bruere, for helpful suggestions.

\title{
BIBLIOGRAPHY.
}

1. Kayser, Centralblatt f. Bakteriol. 1 Pt. vol. 33, page 241.

2. Michalski, Centralbl. f. Bakteriol. Orig. vol. 36, p. 212.

3. Luhenau, Centralbl. f. Bakteriol. Orig. vol. 40, p. 433.

4. Stregulina, Zeitchr, f. Hyg. u. Infektionkrankh. Bd. LI, 1905, p. 18.

5. Charrin and de Nittis. Compt. rendu de la soc. de Biol., 1897, p. 713.

\section{OPHTHALMIC EXAMINATION OF DRAFTED MEN AT CAMP JACKSON.}

Captain Burton Chance, M. D.

\author{
PHILADELPHIA, PA.
}

Ophthalmologist's report covering September to December, 1917. Read before the Section on Ophthalmology of the College of Physicians of Philadelphia, February 21st, 1918.

Up to the middle of December, 1917, the men, white and black, in the first selective draft of the new National Army received at the cantonment at Camp Jackson, Columbia, S. C., were drawn directly from the Carolinas and Florida, but in the later weeks of the period additional men hailing largely from Tennessee and Arkansas, entered by transfer from Camps Pike and Gordon.

As viewed by a stranger from the North, these men presented physical and social characteristics not commonly seen in the ordinary experiences of his practice in an eastern city. Except for the Floridians it might be said that all were descended from families which had been in America for many generations. The white Carolinians were, in the main, above the average height, the majority having long heads, fair complexions; their skins being thin and of delicate texture and their irides blue or gray-characteristics betokening British ancestry, which ancestry was further indicated by their family and given names. The Tennesseeans were not so tall, their facial lines smaller and their complexions swarthy. The Floridians were of mixed types; their names as well as their physical characteristics indicated descent from more recent European emigrant ancestry, while a number were natives of Cuba and of other of the Spanish West Indies.

The negroes were quite African, scarcely any were mulatto. They came from all sections of the states named. They appeared to be members of distinct groups as they could be distinguished by their facial and general physical linea- 
ments, and were further separated by their variations in speech. They were of pure unmixed tribes, apparently, and one fancied that he could tell which were Zulu, which Kaffir, which Hottentot, and which from the Guinea Coast; and, to one conversant with these variations among them it was possible to tell from exactly which county of this state an individual came. A negro from Charleston or the sea coast was readily differentiated from the negroes of the highlands of North and South Carolina. There was a sprinkling of Cherokee and other Indian tribes, long resident in Florida and the Carolinas.

In this present consideration it must be borne in mind that the men sent to the camps by the draft boards were presumed to be in perfect health and quite fit to serve as soldiers; and, that the majority of them did not arrive until several weeks subsequent to their local board examinations. Immediately after arrival at Camp Jackson they were subjected to a rigid examination by the regimental surgeons, before they could be assigned to the various organizations apportioned to the cantonment. When the surgeons were in doubt as to their physical fitness they were referred to a Central Board of Examiners, composed of the chiefs assigned to the several departments of the base hospital, viz., surgery, medicine, including heart, blood vessels and lungs, psychoneurology, venereal disease, otolaryngology and ophthalmology.

It, doubtless, will be of interest to be told of the ophthalmologic affections which were deemed of sufficient gravity to disqualify for the service, according to the regulations prepared by the Surgeon General of the army, and prescribed by the President of the United States, to wit: "The minimum visual requirements to be as follows: $20 / 40$ for the better eye, and $20 / 100$ for the poorer eye, provided that no organic disease exists in either eye." And further: "The following defects are causes for rejection: Acuity of vision below the requirements of the preceding paragraph; conjunctival affections, including trachoma and entropion; strabismus, diseases of the lachrymal apparatus, exophthalmos, ptosis, asthenopia, nystagmus." These require- ments have been greatly modified and will not hold in the future selection of draft men.

Here is a summary of the cases rejected by my board:

Diseases of the Lids and Eyebrows: Marginal blepharitis, 2; chalazion, 1; traumatic coloboma, 1 ; dermoid cyst of the orbital ridge, 1 ; dermatitis venenata, 1 ; distichiasis, 1 ; ectropion after abscess, 2 ; ptosis, 1 ; symblepharon, 1 ; sycosis tarsi, 1.

Lacrimal Apparatus: Dacryocystitis, catarrhal, 1 ; after fracture, 1 ; lacerated wound involving the sac, 1 .

Conjuncrrva: Catarrh, acute, 2; chronic, 8; conjunctivitis, "granular," but not trachomatous, 3 ; distinctly trachomatous, 35 ; gonorrheal, 1 ; gunpowder pigmentation, 1 ; pterygium, unilateral, 2 ; bilateral, 9 ; recurrence after removal by previous operation, 1. The eyelids of every man examined were turned to expose the retrotarsal foldsthe most efficient instrument for the purpose I found to be the corner of the desk blotter, which could be applied to the full length of the tarsus. Later I whittled a stick like a golf stick.

The cases of acute conjunctivitis rejected were marked by symptoms amounting to blennorrhea.

It was not possible for me to ascertain the number of cases of trachomatous disease rejected by the regimental examining surgeons, but the comparatively small number found by the Central Board quite surprised me, as a greater number had been anticipated. The cases seen by us exhibited only moderate signs, not at all so severe as we might have seen at any day's clinic in the northern cities. Few showed cicatrices, however, but all rejected presented roughening of the upper tarsus, with granulation of the free borders and retrotarsal folds. Only two negroes, who were very black and of short stature, had trachoma.

A comparatively large number of cases of specific urethritis arrived at the cantonment, yet only one case of gonorrheal conjunctivitis was referred to me, and he was a negro. The anterior segment of his globe was already destroyed, with the uvea bulging through the corneal perforations, on his arrival. 
In my previous experience I had not seen pterygium in young subjects, yet at these Central Board meetings eleven men were disqualified because the wings extended so far over the corneas as to interfere with sight. One man had bilateral growths, one of which being a recurrence at the site of a mass which had been removed five years previously.

CoRnEa: Nodular degeneration, 2; keratitis, dendritic, 1 ; herpetic, 2 ; interstitial, 1 ; ulceration, 1 ; vascular pannus, 1 ; keratoconus 1 ; leukoma, believed to be malarious, 1 ; maculation, 7 ; gunpowder pigmentation, 1 . Staphyloma, 1; accompanying gonorrheal ophthalmia, 1 ; results of penetrating wounds, 3 .

The cases of nodular degeneration of the cornea were bilateral. In one case the spots were at the centers; in the other they were in the outer zones, yet in each case the peripheries were clear.

It was interesting to have recorded only one case of interstitial keratitis and but a single case of pannus. The endresults of wounds of the cornea showed cicatrices through the limbus with more or less involvement of deeper tissues.

The case presumed to have been malarial keratitis occurred in a man who had had several attacks of malaria in an aggravated form, he stated, in each of which his eyes became inflamed. His last seizure had been within the twelve-month. The inflammation had lasted several weeks; since the subsidence he had noticed that his sight had become imperfect. There was a localized opacity in the parenchyma.

The case of staphyloma was at the upper limbus and had supervened after repeated attacks of inflammation of the anterior segment. It protruded prominently when the lid was raised.

Iris AND Cil.tary Bony: Foreign body in anterior chamber, see glaucoma; ciliary staphyloma, 1 ; coloboma, 2 ; corectopia, 1 ; exclusion of pupil by annular synechia, 1 ; iritis, recurrent, 1 ; ectropion, 1 ; paralysis of spincter pupillae, 1 ; posterior synechia, 2 ; results of lacerated wound, 1. The coloboma of the iris included the sphincter and extended completely into the ciliary body.

AFFECTIONS OF EYEBALL: Exophthalmos, 2; glaucoma, secondary to wounds, with the presence of foreign body in the anterior chamber, 1.

The etiology of the two cases of exophthalmos was not ascertainable; they were not goitrous. In consultations with my colleagues I examined a score of cases, perhaps, of Graves' disease with exophthalmos.

Muscles and Nerves: Nystagmus, horizontal constant, 3; "intermittent," 2. Paralysis: External rectus, 1; inferior oblique, 1; superior rectus, 1 ; strabismus, convergent, 34 ; divergent, 11.

The "intermittent" nystagus exhibited features the like of which I do not recall having seen hitherto. In each case the eyes were quite normal until after slightly prolonged efforts at convergencefixation, when with almost startling rapidity the eyes oscillated from left to right in the chord between the vertical meridian and the extreme right. The movements continued for a few seconds and then after a moments' rest if further efforts at convergence were attempted a similar explosion would follow. I did not consider these to be cases of true nystagmus, but rather that they were due to inherent weakness. Neither man presented signs of generalized nervous disease.

Crystalline Lens: Cataract: Cortical, immature, 1, mature, 2; anterior polar, 3 ; disciformis, 1 ; post-traumatic, 5 ; opacity, 1; results of punctured wound, 1.

Of those showing disorder in the crystalline lens two were cases of mature monocular cataract, in appearance like senile cataract; in each case the fundus of the fellow eye appeared perfectly healthy. The disciform cataract was of the type such as that which I reported some years ago as having found in several members of a family, the opacity being situated behind the nucleus in advance of the posterior pole.

Chorom: Albinism, 1; simple generalized atrophy, 6 ; traumatic atrophy, 1 ; in relation to myopia, 1 ; chorioretinitis, 1 ; associated with acne, 1 ; choroiditis disseminata, 1; coloboma, 1; rupture, 4. The coloboma occupied the lower median fifth, extending up to the edge of the disc. The ruptures were all of the 
usual form in the temporal half of the fundus.

Retina and Optic Nerve: Retinal atrophy, 2; after injury, 1 ; optic atrophy, said to be congenital, probably hereditary, 3 ; postneuritic, 2 ; papilledema, accompanying hemiplegia, 1 ; retinitis proliferans, 3 .

Anomalies of Refraction and AcCommodations: Amblyopia ex anopsia, 5 ; anisometropia, 1 ; hypermetropia, 19 ; myopia, 13 ; paralysis of accommodation by use of cycloplegics, 1 .

Of the nineteen cases of hypermetropia and thirteen myopia rejected, several, who had claimed that they were blind, expressed delight when a manifest correction gave them useful sight.

Malingering: No statistical and analytical records were kept of those who feigned blindness. As already stated, the men had been drawn largely from the country and mountainous districts of the Carolinas and of Florida. There were, also, laborers from the towns of that state. Many received from Tennessee, Kentucky, Arkansas and Mississippi were from districts remote from the large cities. The blacks came chiefly from the Carolinas.

It wats not always easy to ascertain the acuteness of the men's sight, for many could neither read nor write, and not a few did not understand the numeral characters. It should be remembered that the men of greater degrees of education had already enlisterl in the $\mathrm{Na}$ tional fitard. Many magnified their viswal or ocular complaints, yet their efforts at malingering were manifested most crudely. With some it became evident that they had been coached. because I noticed that they would rearlily read to. say, 20/70, with the right eye, and $20 / 100$ or $20 / 200$. with the left, yet, while oft their guard, when the order of examination was reversed, would read better with the claimed-to-be-poor eye and worse with the hitherto good eye. Others lost control of themselves by their interest in pointing the direction in which the arms of the "illiterate- E" were placed while others were detected by their ability to count the strokes of those of the $20 / 15$ line; and others perceived short 1 -inch lines which they counted at full
20/20. Quite a number deliberately closed their eyes, leclaring they could not see; others feigned complete blindness, yet they admitted that they had for years followed gainful handicrafts and that they had found their way unaided from their barracks, to which they had heen introduced in the mitdle of the night previous. Others, whose manifested standards were below requirements, evinced the greatest interest in the records while they were being made, and, several were detected reading the cards with the greatest ease.

Among the inflammatory states seen were several cases which I assumed to have been self-inflicted, as, for instance, by the placing of gritty sulbstances beneath the eyelids. Such inflammations were always monocular and the physical signs localized. One individual had complete long continued mydriasis with $\mathrm{cy}^{-}$ cloplegia artificially inducerl. No man tried to simulate blindness who had actual disease of his eyes or lids likely to destroy his sight.

Every man referred to me was given a dark room examination. A common complaint by those whose visual acuteness was under question was that the reflection from the ophthalmoscope wats painful and blinded them. In the tests prisms, stereoscopes and colored glasses were used. My methods of procedure sought to analyze the history of each man's sight, and, in addition to the inspection of his cyes, a survey of the general health in needful cases was made.

My service on the board became a delightful experience, the members cooperated with ever-ready helpfulness, and, while every effort was made to accomplish the day's quota with dispatch, time was taken to study each man's case from every point of view for the proper rejection of ineligible men.

I here wish to pay tribute to my colleagues. All had been called from active practice and in the day's round they gave of their best. Our service on the "Central Board" fitted us for a still closer association in the wards of the base hospital when it was opened for the reception of the sick. Of my experiences in the hospital I trust to be allowed to speak at another time. 